\section{To Drop or Not to Drop: Design Principles for Kalman Filtering Over Wireless Fading Channels}

\author{
Yasamin Mostofi, Member, IEEE, and \\ Richard M. Murray, Fellow, IEEE
}

\begin{abstract}
It is the general assumption that in estimation and control over wireless links, the receiver should drop any erroneous packets. While this approach is appropriate for non real-time data-network applications, it can result in instability and loss of performance in networked control systems. In this technical note we consider estimation of a multiple-input multiple-output dynamical system over a mobile fading communication channel using a Kalman filter. We show that the communication protocols suitable for other already-existing applications like data networks may not be entirely applicable for estimation and control of a rapidly changing dynamical system. We then develop new design paradigms in terms of handling noisy packets for such delay-sensitive applications. We reformulate the estimation problem to include the impact of stochastic communication noise in the erroneous packets. We prove that, in the absence of a permanent cross-layer information path, packet drop should be designed to balance information loss and communication noise in order to optimize the performance.
\end{abstract}

Index Terms-Estimation over wireless links, fading channels, networked control systems.

\section{INTRODUCTION}

Recently there has been considerable interest in estimation and control over wireless links. Advances in technology have resulted in an abundance of cheap embedded units equipped with sensing, processing, communication and actuation capabilities. This has resulted in a wide range of sensor network applications from environmental monitoring, emergency response, smart homes and factories to surveillance, security and military [1]. Such applications bring together different aspects of estimation, communication and control, necessitating non-traditional and cross-disciplinary designs and approaches.

In this technical note, we are interested in mobile sensor networks that are running real-time applications and are therefore delay-sensitive. We consider a mobile sensor that is observing a dynamical system. It transmits its observation over a wireless link to a remote node that is in charge of estimation using a Kalman filter. This is a fundamental problem that can arise in networked sensing, estimation and control. Communication plays a key role in the overall performance of such networks since both sensor measurements and control commands are transmitted over wireless links. Considering the impact of communication channels on estimation and control is an emerging area of research and has recently been cited as one of the major challenges of the control area [2]. Digital transmission over wireless links can experience bit error rate due to multipath fading, shadowing, receiver thermal noise and excessive distance between the transmitter and receiver. This is in addition to the impact of quantization.

Manuscript received August 24, 2006; revised September 26, 2007 and May 20, 2008. Current version published February 11, 2009. This technical note was presented in part at ACC'07. Recommended by Associate Editor S. Dey.

Y. Mostofi is with the University of New Mexico, Albuquerque, NM 87131 USA (e-mail: ymostofi@ece.unm.edu).

R. M. Murray is with the California Institute of Technology, Pasadena, CA 91125 USA.

Color versions of one or more of the figures in this technical note are available online at http://ieeexplore.iee.org.

Digital Object Identifier 10.1109/TAC.2008.2008331
While impact of quantization on estimation and control over communication links has received considerable attention [3], the impact of other channel unreliability such as fading, shadowing, receiver thermal noise and mobility has not been studied extensively. For mobile applications, such channel unreliability will introduce a time-varying noise in the received samples. The receiver can then decide to either keep the received sample or drop it. The criteria for making this decision vary depending on the application. Data networks, for example, are not as sensitive to delays since the application is not real time. The receiver, therefore, can afford to drop erroneous packets and wait for retransmission. Voice applications such as cellular networks, on the other hand, are sensitive to delays. In every transmitted bit stream, there are key bits embedded for synchronization and other crucial tasks. If these bits get corrupted, the receiver drops the transmitted stream. However, once these bits are received accurately, the rest of the bit error rate is either corrected through channel coding or tolerated [4] since there is no time for retransmission. Estimation and control of dynamical systems over wireless links is an emerging application, for which new communication design paradigms should be developed. Control applications are typically delay sensitive as we are racing against the dynamics of the system. Therefore, the communication protocols and designs suitable for other already-existing applications like data networks may not be entirely applicable.

Current work in literature has assumed applying data network design principles to networked control applications by considering a receiver that only keeps noise-free packets. Along this line, impact of packet drop on networked control applications has been studied. Micheli et al. investigated impact of packet loss on estimation by considering random sampling of a dynamical system [5]. This is followed by the work of Sinopoli et al. which derived bounds for the maximum tolerable probability of packet loss to maintain stability [6]. Impact of the communication noise introduced by excessive distance between the transmitter and receiver, fading, shadowing and mobility, however, has not been studied extensively. Furthermore, an investigation of the appropriate receiver design approach in terms of handling noisy samples is lacking.

In this technical note we are interested in studying the impact of the unreliability introduced by multipath fading channels on estimation. Instead of applying data network design principles to such delay-sensitive applications, we are interested in finding new design paradigms. Inspired by delay-sensitive voice applications, we take a fundamentally different approach and formulation which will allow us to provide the right abstraction for modeling the impact of stochastic communication noise in these systems. The main question this technical note addresses is the following: "How should noisy packets be handled in the receiver?" We answer this question both in terms of stability and performance.

\section{SYSTEM MODEL}

Consider a mobile sensor observing a system with the following linear dynamics:

$$
\begin{aligned}
x[k+1] & =A x[k]+w[k] \\
y[k] & =C x[k]+v[k]
\end{aligned}
$$

where $x[k] \in \mathbb{R}^{N}$ and $y[k] \in \mathbb{R}^{M}$ represent the state and observation respectively. $w[k] \in \mathbb{R}^{N}$ and $v[k] \in \mathbb{R}^{M}$ represent zero-mean Gaussian process and observation noise vectors with covariances of $Q \succeq 0$ and $R \succ 0$ respectively. In this technical note, we take $M=N$ 
and $C$ invertible to focus on the impact of communication noise. We are interested in estimating unstable dynamics and therefore we consider cases where matrix $A$ has at least one eigenvalue outside the unit circle. The sensor then transmits its observation over a wireless fading channel to a remote node, which is in charge of estimation. Since estimation of dynamical systems over mobile links has not been extensively studied before, we keep our analysis general by considering mobile channels.

\section{A. Physical Layer: Wireless Communication [7]}

In this part we will summarize how to model the impact of a timevarying fading wireless communication channel on the observation. The sensor quantizes the observation, $y[k]$, transforms it into a packet of bits and transmits it over a fading channel. The remote node will receive a noisy version of the transmitted data due to bit flip. Let $\hat{y}[k]$ represent the received signal. $\hat{y}[k]$ is what the second node assumes the $k^{t h}$ transmitted observation was. Let $n[k]$ represent the difference between the transmitted observation and the received one: $n[k]=y[k]-\hat{y}[k]$, where $n[k]=n_{c}[k]+n_{q}[k]$. In this technical note, we refer to $n[k]$ as communication noise. It consists of two parts, link noise $\left(n_{c}\right)$ : noise due to the quality of the communication link and quantization noise $\left(n_{q}\right)$. For fading channels, the impact of link noise typically dominates the impact of quantization noise [7]. However, while estimation/control in the presence of quantization noise has received considerable attention, impact of fading on estimation and control has mainly remained unexplored. Therefore, in this technical note we will mainly focus on the impact of the link noise.

1) Multipath Fading Channel: One of the major performance degradation factors of a mobile communication environment is multipath fading. "Multipath" is a term used to describe multiple paths that a radio wave may follow between the transmitter and the receiver. Waves that are received in phase reinforce each other producing a stronger signal, while those that are received out of phase produce a weaker signal. Small changes in the transmission paths, caused by movements of the receiver or transmitter can change the phase relationship of the two signals, introducing a rapidly time-varying fading channel. This is in addition to the distance-dependent attenuation factor. Signal attenuation and fading can result in bit error rate, i.e. some of the transmitted bits will be flipped. This will result in an erroneous reception of the transmitted packets, i.e. $n_{c}[k] \neq 0$. Correlation characteristics of fading channels depend on several parameters such as the transmission environment, speed of the mobile unit, and frequency of operation. For instance, for a mobile node that moves at $25 \mathrm{mph}$ and communicates at $1 \mathrm{GHz}$, channel will be uncorrelated after $13.5 \mathrm{~ms}$ using Jakes model [7]. Therefore, as long as the time interval between consecutive transmissions is larger than $13.5 \mathrm{~ms}$ in a networked control setup, channel can be considered uncorrelated from one transmission to the next. This time interval corresponds to observing a dynamical system at $74 \mathrm{~Hz}$. As the mobile speed or frequency of operation increases, the channel gets uncorrelated even faster. Therefore, in this technical note we take the channel to be uncorrelated from one transmission to the next as it will be the case for several networked control applications.

2) Channel Signal to Noise Ratio: A fundamental parameter that characterizes the performance of a communication channel is the received Signal to Noise Ratio. Received Signal to Noise Ratio is defined as the ratio of the received signal power divided by the receiver thermal noise power. Let $\Upsilon[k]$ represent the instantaneous received Signal to Noise Ratio at $k^{t h}$ transmission. $\Upsilon[k]$ determines how well the transmitted bits of the $k^{\text {th }}$ transmission can be retrieved. As the sensor moves, the remote node will experience different channels and therefore different received Signal to Noise Ratios. In a given area, $\Upsilon[k]$ can be considered a stationary stochastic process with $\Upsilon_{\text {ave }}$ representing its average. The distribution of $\Upsilon[k]$ is a function of the transmission environment and the level of mobility of the sensor. In this technical note we do not make any assumption on the probability distribution of $\Upsilon$. Only when we want to provide an example, we will take $\Upsilon$ to be exponentially distributed, which is a common model for outdoor fading channels with no Line-of-Sight paths.

3) Communication Noise Variance: Let $\sigma_{n}^{2}[k]$ represent the variance of $n[k]$ at $k^{t h}$ transmission. $\sigma_{n}^{2}[k]$ is a function of $\Upsilon[k]$ :

$$
\sigma_{n}^{2}[k]=\mathbb{E}\left(n^{2}[k] \mid \Upsilon[k]\right)=\Xi(\Upsilon[k])
$$

where $\Xi$ is a non-increasing function that depends on the transmitter and receiver design principles, such as modulation and coding, as well as the transmission environment. To keep our analysis general, in this technical note we do not make any assumption on $\Xi$. It should be noted that the communication noise has a time-varying variance.

4) Packet Drop Probability: Depending on the receiver design, there can be a packet drop mechanism deployed in the receiver. Let $\mu[k]$ represent the probability that the receiver drops the $k^{t h}$ packet. $\mu[k]$ can also be represented as a function of $\Upsilon[k]: \mu[k]=G(\Upsilon[k])$, where function $G$ is a non-increasing function. Functions $\Xi$ and $G$ provide the abstraction necessary to model the impact of the physical layer in the higher application layer. Experimental results have shown $G$ to be well approximated as follows [8]:

$$
\mu[k]= \begin{cases}0 & \Upsilon[k] \geq \Upsilon_{T} \\ 1 & \text { else }\end{cases}
$$

This means that the receiver keeps those packets with the received instantaneous Signal to Noise Ratio above a designated threshold $\Upsilon_{T}$.

\section{B. Application Layer: Estimation}

The remote node estimates the state based on the received observation using a Kalman filter [9]. Let $\hat{x}[k]$ denote the estimate of $x[k]$ at the remote node. Then $P[k]$ represents the corresponding estimation error covariance matrix given $\Upsilon[k-1], \Upsilon[k-2], \ldots, \Upsilon[0]$ :

$$
P[k]=\mathbb{E}\left[(x[k]-\hat{x}[k])(x[k]-\hat{x}[k])^{T}\right]_{\mid \Upsilon[k-1], \Upsilon[k-2], \ldots, \Upsilon[0]} .
$$

This is different from the traditional form of Kalman filtering since $P[k]$ is a function of channel statistics through $\Upsilon[k-1], \Upsilon[k-$ $2], \ldots, \Upsilon[0]$. For instance, to obtain $\mathbb{E}(P[k]), P[k]$ should be averaged over the joint distribution of $\Upsilon[k-1], \Upsilon[k-2], \ldots, \Upsilon[0]$.

\section{Cross-Layer Information Path}

It is well known that data networks enjoy a layered protocol stack that makes different layers opaque to one another. When estimating over wireless links, the application layer will be in charge of estimation whereas the knowledge of the quality of the communication link will be available in the physical layer. A cross-layer information path in this case refers to a path from the physical layer to the application layer that carries information on the quality of the link (Signal to Noise Ratio or communication noise variance). Since mobile fading channels can decorrelate and change rapidly, there needs to be a constant cross-layer information path to update the application layer of the link quality. While the presence of such a path can play a key role in the overall 
performance, the receiver architecture may not support it. Therefore, in this technical note we will consider scenarios where such a path is not available in the receiver, i.e. the application layer does not have any knowledge on the link quality.

\section{Ideal Communication Noise}

As discussed in Section I, current work in literature mainly applies data network design principles to networked control applications by assuming that the receiver drops packets that contain any amount of error. Then those packets that are kept in the receiver are considered noise-free. We refer to this assumption on the communication noise as "ideal noise" throughout the technical note. Such an assumption translates to the following recursion for the estimation error covariance:

$$
\begin{aligned}
& P[k+1]=A P[k] A^{T}+Q \\
& \quad-A P[k] C^{T}\left(R+C P[k] C^{T}+S_{1}(\Upsilon[k])\right)^{-1} C P[k] A^{T}
\end{aligned}
$$

where

$$
S_{1}[k]= \begin{cases}0 & \Upsilon[k] \geq \Upsilon_{T} \\ \infty & \text { otherwise }\end{cases}
$$

Current work in literature mainly considers Kalman filtering over fixed wireless channels by assuming that the probability of packet drop is the same from one transmission to the next. For a fixed probability of packet drop, $\mu_{\text {fixed }}$, authors in [6] found the following condition for stability: ${ }^{1}$

$$
\mu_{\text {fixed }}<\rho_{\max }^{-2}
$$

where $\rho_{\max }$ represents spectral radius of matrix $A$.

\section{RECEIVER DESIGN THEORIES}

Estimation of a rapidly changing dynamical system is delay sensitive. Dropping all the erroneous packets can result in loss of information, can reduce the useful transmission rate and can lead to instability for such applications. Therefore, the receiver can not afford to wait for receiving noise-free packets. In this section, we will consider the impact of stochastic communication noise on the estimation of a multiple-input multiple-output system and will derive a new receiver design strategy in terms of handling the received information, which is more suitable for real-time networked control applications. To keep the analysis general, we will not make any assumption on the communication noise variance function or Signal to Noise Ratio distribution. To ease mathematical derivation, we assume that the observation noise is negligible compared to the communication noise. The estimation using a Kalman filter will then be as follows:

$$
\hat{x}[k+1]= \begin{cases}A \hat{x}[k] & \text { if } k^{t h} \text { packet is dropped } \\ A C^{-1} y[k] & \text { if } k^{t h} \text { packet is kept. }\end{cases}
$$

The estimation error will be as follows using (1):

$$
\begin{aligned}
x[k+1] & -\hat{x}[k+1] \\
= & \begin{cases}A(x[k]-\hat{x}[k])+w[k] & \text { if } k^{t h} \text { packet is dropped } \\
w[k]-A C^{-1} v[k] & \text { if } k^{t h} \text { packet is kept. }\end{cases}
\end{aligned}
$$

${ }^{1}$ Note that the original result of [6] is for a general $C$ matrix and results in (7) when $C$ is invertible.
This will result in the following recursion for the estimation error covariance:

$$
\begin{aligned}
P[k+1]=A P[k] & A^{T}+Q \\
& -\frac{A P[k] A^{T}-\sigma_{n}^{2}(\Upsilon[k]) A\left(C^{T} C\right)^{-1} A^{T}}{S_{2}[k]}
\end{aligned}
$$

where $\sigma_{n}^{2}$ is the communication noise variance as defined in Section II and

$$
S_{2}[k]= \begin{cases}1 & \Upsilon[k] \geq \Upsilon_{T} \\ \infty & \text { otherwise }\end{cases}
$$

As the mobile node moves in a given area, it will experience different Signal to Noise Ratios. Averaging (10) over $\Upsilon[k]$ will result in the following recursion for average estimation error covariance:

$$
\begin{aligned}
\mathbb{E}(P[k+1])=\mu_{\text {ave }}\left(\Upsilon_{T}\right) A \mathbb{E}( & P[k]) A^{T}+Q \\
& +\sigma_{n, a v e}^{2}\left(\Upsilon_{T}\right) A\left(C^{T} C\right)^{-1} A^{T} .
\end{aligned}
$$

$\mu_{\text {ave }}$ and $\sigma_{n, \text { ave }}^{2}$ represent average probability of packet loss (spatial averaging) and average communication noise variance that entered the estimation process respectively:

$$
\mu_{\text {ave }}\left(\Upsilon_{T}\right)=\mathbb{E}(\mu)=\int_{0}^{\Upsilon_{T}} \chi(\Upsilon) d \Upsilon
$$

and

$$
\sigma_{n, \text { ave }}^{2}\left(\Upsilon_{T}\right)=\int_{\Upsilon_{T}}^{\infty} \sigma_{n}^{2}(\Upsilon) \chi(\Upsilon) d \Upsilon
$$

where $\chi$ represents probability density function of $\Upsilon$.

Lemma 1 (See [9]): Consider the following Lyapunov equation with $\Theta$ Hermitian: $\Sigma=\Pi \Sigma \Pi^{T}+\Theta$. The following hold:

a) If $\Pi$ is a stable matrix (spectral radius less that one), $\Sigma$ will be unique and Hermitian and can be expressed as follows: $\Sigma=$ $\sum_{i=0}^{\infty} \Pi^{i} \Theta\left(\Pi^{T}\right)^{i}$,

b) if $\left\{\Pi, \Theta^{1 / 2}\right\}$ is controllable and $\Theta \succeq 0$, then $\Sigma$ will be Hermitian, unique and positive-definite iff $\Pi$ is stable.

\section{A. Stability}

Definition 1: We consider the estimation process stable as long as average estimation error covariance stays bounded.

Using Lemma $1 b$, it can be easily seen from (12) that the stability condition will be as follows:

$$
\mu_{\text {ave }}<\rho_{\max }^{-2}
$$

where $\rho_{\max }$ represents the spectral radius of matrix $A$.

Remark 1: The stability condition is independent of the shape of the communication noise variance, $\sigma_{n}^{2}$.

Remark 2: Define stability range as the range of all the $A$ matrices or average Signal to Noise Ratios, $\Upsilon_{a v e}$, that would satisfy the stability condition of (15). It can be easily confirmed that decreasing $\Upsilon_{T}$ (keeping more packets) will increase the stability range. 
While keeping all the packets results in maximizing the stability range, it will not minimize the estimation error covariance. Next we show how to design the receiver to minimize the estimation error covariance.

\section{B. Optimum Performance}

Intuitively, there should be an optimum $\Upsilon_{T}$ (optimum way of dropping packets) that will minimize the asymptotic average estimation error covariance. If $\Upsilon_{T}$ is too low, the receiver will keep most of the packets but the estimation will be too noisy. On the other hand, if $\Upsilon_{T}$ is too high, the receiver will be strict about the quality of the packets that it will keep. This reduces the amount of communication noise that enters the estimation process but will result in high packet loss rate and therefore information loss rate. Then the optimum $\Upsilon_{T}$ will be the one that provides a balance between information loss and communication noise. The asymptotic average estimation error covariance will be as follows as long as the stability condition of (15) holds:

$$
\begin{aligned}
\mathbb{E}(P[\infty])= & \mu_{\text {ave }}\left(\Upsilon_{T}\right) A \mathbb{E}(P[\infty]) A^{T}+\sigma_{n, \text { ave }}^{2}\left(\Upsilon_{T}\right) A \\
& \times\left(C^{T} C\right)^{-1} A^{T}+Q \text { for } \mu_{\text {ave }}\left(\Upsilon_{T}\right)<\rho_{\max }^{-2} .
\end{aligned}
$$

Let $\Upsilon_{T_{1} \text { opt }}$ represent the optimum way of dropping packets which will minimize the spectral norm of the asymptotic average estimation error covariance matrix: $\Upsilon_{T_{1}, \text { opt }}=\arg \min \left\|\mathbb{E}\left(P\left[\infty, \Upsilon_{T}\right]\right)\right\|$. Let $\Upsilon_{T_{2}, \text { opt }}$ represent the optimum way of dropping packets which will minimize the determinant of the asymptotic average estimation error covariance: $\Upsilon_{T_{2} \text { opt }}=\arg \min \operatorname{det} \mathbb{E}\left(P\left[\infty, \Upsilon_{T}\right]\right)$. We will derive an analytical expression that relates optimum way of dropping packets to the characteristics of the communication channel and eigenvalues of matrix $A$. For this derivation, we assume that $C=\varsigma I_{N}$ and $Q=q I_{N}$, where $I_{N}$ represents an $N \times N$ identity matrix. We furthermore take $A=A_{s}$, where $A_{s}$ is a symmetric matrix, i.e. $A_{s}=A_{s}^{T}$. Later in this section we will discuss cases where $A$ is not symmetric.

Theorem 1 (Balance of Information Loss \& Communication Noise): Consider the system model described in Section II, with $C=\varsigma I_{N}$, $Q=q I_{N}$ and $A=A_{s}$. Consider a receiver that is equipped with a packet drop mechanism described by (3) and does not support a crosslayer path. Then $\Upsilon_{T_{1} \text {,opt }}$ will be as follows:

$$
\Upsilon_{T_{1}, \text { opt }}= \begin{cases}\Upsilon_{T_{1}}^{*} & \Upsilon_{T_{1}}^{*} \geq 0 \\ 0 & \text { otherwise }\end{cases}
$$

where $\Upsilon_{T_{1}}^{*}$ is the unique solution to the following equation:

$$
\begin{aligned}
\underbrace{\mu_{\text {ave }}\left(\Upsilon_{T_{1}}^{*}\right)}_{\text {information loss }}+\underbrace{\sigma_{n, \text { norm }}^{2}\left(\Upsilon_{T_{1}}^{*}\right)}_{\text {communication noise }}+ \\
\frac{\varsigma^{2} q}{\rho_{\max }^{2} \sigma_{n}^{2}\left(\Upsilon=\Upsilon_{T_{1}}^{*}\right)}=\rho_{\max }^{-2}
\end{aligned}
$$

where $\sigma_{n, \text { norm }}^{2}$ refers to the normalized average communication noise variance: $\sigma_{n, \text { norm }}^{2}\left(\Upsilon_{T_{1}}^{*}\right)=\sigma_{n, \text { ave }}^{2}\left(\Upsilon_{T_{1}}^{*}\right) / \sigma_{n}^{2}\left(\Upsilon=\Upsilon_{T_{1}}^{*}\right)$, and $\Upsilon_{T_{2} \text {,opt }}$ will be as follows:

$$
\Upsilon_{T_{2}, \text { opt }}= \begin{cases}\Upsilon_{T_{2}}^{*} & \Upsilon_{T_{2}}^{*} \geq 0 \\ 0 & \text { otherwise }\end{cases}
$$

where $\Upsilon_{T_{2}}^{*}$ is the unique solution to the following equation:

$$
\sum_{i=1}^{N} \frac{\rho_{i}^{2}}{1-\rho_{i}^{2} \mu_{\text {ave }}\left(\Upsilon_{T_{2}}^{*}\right)}=\sum_{i=1}^{N} \frac{1}{\sigma_{n, \text { norm }}^{2}\left(\Upsilon_{T_{2}}^{*}\right)+\frac{q \varsigma^{2}}{\sigma_{n}^{2}\left(\Upsilon=\Upsilon_{T_{2}}^{*}\right) \rho_{i}^{2}}}
$$

where $\rho_{1}, \rho_{2}, \ldots, \rho_{N}$ represent the eigenvalues of matrix $A$, where $\left|\rho_{1}\right| \geq\left|\rho_{2}\right| \geq \ldots \geq\left|\rho_{N}\right|$ and $\rho_{\max }=\left|\rho_{1}\right|$.

Proof of Theorem 1: Using Lemma la with $C=\varsigma I_{N}$, $Q=q I_{N}$ and $A=A_{s}$ will result in $\mathbb{E}(P[\infty])=$ $\varsigma^{-2} \sigma_{n, a v e}^{2}\left(\Upsilon_{T}\right) \sum_{i=0}^{\infty} \mu_{a v e}^{i}\left(\Upsilon_{T}\right)\left(A_{s}\right)^{2 i+2}+q \sum_{i=0}^{\infty} \mu_{\text {ave }}^{i}\left(\Upsilon_{T}\right)\left(A_{s}\right)^{2 i}$. We will have the following decomposition: $A_{s}=L \Lambda L^{T}$, where $\Lambda=\operatorname{diag}\left\{\rho_{1}, \rho_{2}, \ldots, \rho_{N}\right\}$ and $L^{T} L=I_{N}$. It can be confirmed that

$$
\begin{aligned}
& \mathbb{E}(P[\infty])= \\
& L \times \operatorname{diag}\left\{\frac{q+\varsigma^{-2} \rho_{1}^{2} \sigma_{n, a v e}^{2}\left(\Upsilon_{T}\right)}{1-\rho_{1}^{2} \mu_{\text {ave }}\left(\Upsilon_{T}\right)}, \ldots, \frac{q+\varsigma^{-2} \rho_{N}^{2} \sigma_{n, a v e}^{2}\left(\Upsilon_{T}\right)}{1-\rho_{N}^{2} \mu_{\text {ave }}\left(\Upsilon_{T}\right)}\right\} L^{T}
\end{aligned}
$$

where $\operatorname{diag}\{\}$ denotes a diagonal matrix with the elements inside the parenthesis representing the diagonal elements. This results in

$$
\|\mathbb{E}(P[\infty])\|=\left(\frac{q+\varsigma^{-2} \rho_{1}^{2} \sigma_{n, a v e}^{2}\left(\Upsilon_{T}\right)}{1-\rho_{1}^{2} \mu_{a v e}\left(\Upsilon_{T}\right)}\right) .
$$

Let $\Upsilon_{T_{1}}^{*}$ represent any solution to (18). It can be easily verified that $\partial E(\|P[\infty]\|) / \partial \Upsilon_{T}$ is only zero at $\Upsilon_{T_{1}}^{*}$. Next we show that (18) has a unique solution. Assume that (18) has two solutions: $\Upsilon_{T_{1,1}}^{*}$ and $\Upsilon_{T_{1,2}}^{*}>\Upsilon_{T_{1,1}}^{*}$. Since $\sigma_{n}^{2}$ is a non-increasing function of $\Upsilon$, we will have the following:

$$
\begin{aligned}
& \mu_{\text {ave }}\left(\Upsilon_{T_{1,1}}^{*}\right)+\sigma_{n, \text { norm }}^{2}\left(\Upsilon_{T_{1,1}}^{*}\right)+\frac{\varsigma^{2} q}{\rho_{\max }^{2} \sigma_{n}^{2}\left(\Upsilon=\Upsilon_{T_{1,1}}^{*}\right)} \\
& -\left[\mu_{\text {ave }}\left(\Upsilon_{T_{1,2}}^{*}\right)+\sigma_{n, \text { norm }}^{2}\left(\Upsilon_{T_{1,2}}^{*}\right)+\frac{\varsigma^{2} q}{\rho_{\max }^{2} \sigma_{n}^{2}\left(\Upsilon=\Upsilon_{T_{1,2}}^{*}\right)}\right] \\
& =\underbrace{\int_{\Upsilon_{T_{1,2}}^{*}}^{\Upsilon_{T_{1,1}}^{*}} \chi(\Upsilon) d \Upsilon+\int_{\Upsilon_{T_{1,1}}^{*}}^{\Upsilon_{T_{1,2}}^{*}} \frac{\sigma_{n}^{2}(\Upsilon) \chi(\Upsilon)}{\sigma_{n}^{2}\left(\Upsilon=\Upsilon_{T_{1,1}}^{*}\right)} d \Upsilon}_{<0} \\
& +\underbrace{\left(\frac{1}{\sigma_{n}^{2}\left(\Upsilon=\Upsilon_{T_{1,1}}^{*}\right)}-\frac{1}{\sigma_{n}^{2}\left(\Upsilon=\Upsilon_{T_{1,2}}^{*}\right)}\right) \int_{\Upsilon_{T_{1,2}^{*}}^{*}}^{\infty} \sigma_{n}^{2}(\Upsilon) \chi(\Upsilon) d \Upsilon}_{<0} \\
& +\underbrace{\frac{\varsigma^{2} q}{\rho_{\max }^{2}}\left(\frac{1}{\sigma_{n}^{2}\left(\Upsilon=\Upsilon_{T_{1,1}}^{*}\right)}-\frac{1}{\sigma_{n}^{2}\left(\Upsilon=\Upsilon_{T_{1,2}}^{*}\right)}\right)}_{<0}<0 .
\end{aligned}
$$

Therefore, ${ }^{2} \Upsilon_{T_{1,1}}^{*}=\Upsilon_{T_{1,2}}^{*}$. Let $\Upsilon_{T}^{c}$ be the critical stability threshold: $1-\rho_{\text {max }}^{2} \mu_{\text {ave }}\left(\Upsilon_{T}^{c}\right)=0$. We have $\Upsilon_{T_{1}}^{*}<\Upsilon_{T}^{c}$. Consider those cases where there exists a positive solution to (18). Then using the fact that $\lim _{\Upsilon_{T} \rightarrow \Upsilon_{T}^{c}} \mathbb{E}\left(P\left[\infty, \Upsilon_{T}\right]\right) \rightarrow \infty$ shows that $\Upsilon_{T_{1}}^{*}$ corresponds to the unique minimum of $\left\|\mathbb{E}\left(P\left[\infty, \Upsilon_{T}\right]\right)\right\|$, i.e. $\Upsilon_{T_{1}, o p t}=\Upsilon_{T_{1}}^{*}$. If process noise is the dominant noise, compared to the communication noise, there may be no positive solution to (18). It can be easily seen that, in such cases, $\left\|\mathbb{E}\left(P\left[\infty, \Upsilon_{T}\right]\right)\right\|$ will be an increasing function for $\Upsilon_{T} \geq$ 0 , resulting in $\Upsilon_{T_{1}, o p t}=0$.

Next we will find $\Upsilon_{T_{2} \text {,opt }}$. We will have

$$
\operatorname{det} \mathbb{E}(P[\infty])=\prod_{i=1}^{N} \frac{\rho_{i}^{2} \varsigma^{-2} \sigma_{n, \text { ave }}^{2}\left(\Upsilon_{T}\right)+q}{1-\rho_{i}^{2} \mu_{\text {ave }}\left(\Upsilon_{T}\right)} .
$$




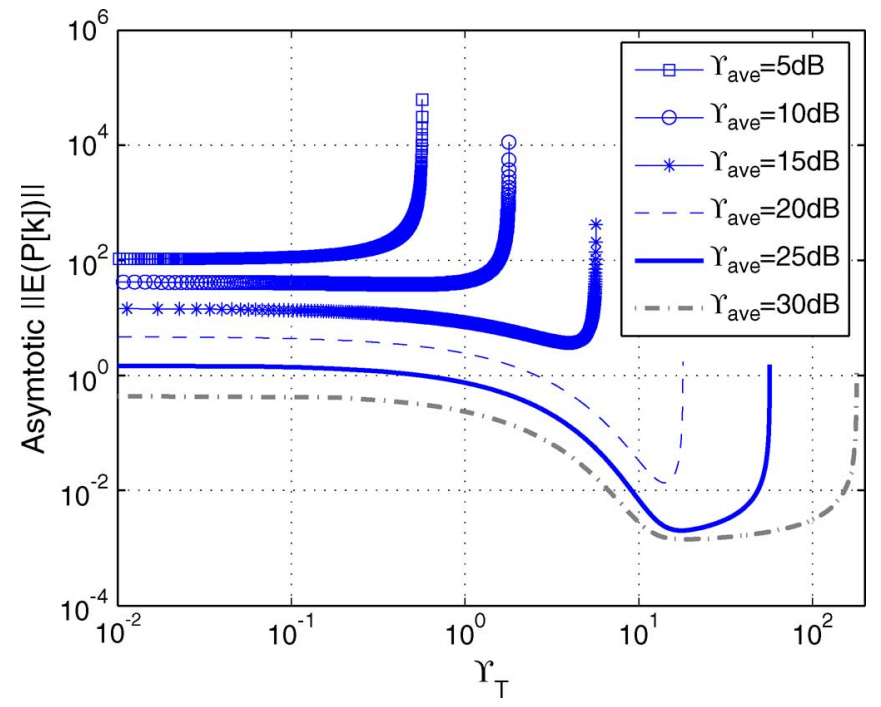

Fig. 1. Minimums of the curves indicating the optimum packet drop mechanism.

It can be easily confirmed that

$$
\begin{aligned}
& \frac{\partial \operatorname{det} \mathbb{E}(P[\infty])}{\partial \Upsilon_{T}}=\chi(\Upsilon) \frac{\prod_{i=1}^{N}\left(\varsigma^{-2} \sigma_{n, a v e}^{2}\left(\Upsilon_{T}\right) \rho_{i}^{2}+q\right)}{\prod_{i=1}^{N}\left(1-\rho_{i}^{2} \mu_{\text {ave }}\left(\Upsilon_{T}\right)\right)} \\
& \times\left[\sum_{j=1}^{N} \frac{\rho_{j}^{2}}{1-\rho_{j}^{2} \mu_{\text {ave }}\left(\Upsilon_{T}\right)}-\sum_{j=1}^{N} \frac{\varsigma^{-2} \sigma_{n, a v e}^{2}\left(\Upsilon_{T}\right) \rho_{j}^{2}}{\varsigma^{-2} \sigma_{n, a v e}^{2}\left(\Upsilon_{T}\right) \rho_{j}^{2}+q}\right] .
\end{aligned}
$$

Therefore, $\partial \operatorname{det}(\mathbb{E}(P[\infty])) /\left.\partial \Upsilon_{T}\right|_{\Upsilon_{T}=\Upsilon_{T_{2}}^{*}}=0$ will result in (20). In a similar manner, it can be easily confirmed that (20) has a unique solution and that $\Upsilon_{T_{2} \text { opt }}$ corresponds to the global minimum of the determinant of the asymptotic average estimation error covariance.

Theorem 1 shows that the optimum way of dropping packets is the one that provides a balance between information loss $\left(\mu_{\text {ave }}\right)$ and communication noise $\left(\sigma_{n, a v e}^{2}\right)$. Equation (18) [and (20)] may not have a positive solution if process noise is the dominant noise compared to the communication noise (the third term on the left hand side of (18), for instance, will then get considerably high values). In such cases, the receiver should keep all the packets as communication noise is not the bottleneck. However, as long as process noise is not the dominant noise, the optimum way of dropping packets is the one that provides a balance between information loss and communication noise as indicated by (18) [and (20)].

Theorem 1 confirms that dropping all the erroneous packets will not minimize the estimation error covariance and that the optimum receiver would allow some amount of communication noise in the estimation process in order to avoid high information loss rate. In general, minimizing spectral norm and determinant could result in different optimum packet drop thresholds depending on the eigenvalues of matrix $A$, as can be seen from Theorem 1. If $A$ has one dominant eigenvalue or all the eigenvalues of $A$ are the same, then $\Upsilon_{T_{1} \text { opt }}=\Upsilon_{T_{2} \text {,opt }}$. To see this, note that (18) can be written as

$$
\frac{\rho_{\text {max }}^{2}}{1-\rho_{\text {max }}^{2} \mu_{\text {ave }}\left(\Upsilon_{T_{1}}^{*}\right)}=\frac{1}{\sigma_{n, \text { norm }}^{2}\left(\Upsilon_{T_{1}}^{*}\right)+\frac{q^{2}}{\sigma_{n}^{2}\left(\Upsilon=\Upsilon_{T_{1}}^{*}\right) \rho_{\text {max }}^{2}}}
$$

Therefore, if all the eigenvalues are the same, (18) and (20) will be the same. On the other hand, if $\rho_{\max }$ is the dominant eigenvalue of $A$, we will have

$$
\sum_{i=1}^{N} \frac{\rho_{i}^{2}}{1-\rho_{i}^{2} \mu_{\text {ave }}\left(\Upsilon_{T_{2}}^{*}\right)} \approx \frac{\rho_{\max }^{2}}{1-\rho_{\max }^{2} \mu_{\text {ave }}\left(\Upsilon_{T_{2}}^{*}\right)}
$$

and

$$
\begin{aligned}
& \sum_{i=1}^{N} \frac{1}{\sigma_{n, \text { norm }}^{2}\left(\Upsilon_{T_{2}}^{*}\right)+\frac{q \varsigma^{2}}{\sigma_{n}^{2}\left(\Upsilon=\Upsilon_{T_{2}}^{*}\right) \rho_{i}^{2}}} \\
& \approx \frac{1}{\sigma_{n, \text { norm }}^{2}\left(\Upsilon_{T_{2}}^{*}\right)+\frac{q \varsigma^{2}}{\sigma_{n}^{2}\left(\Upsilon=\Upsilon_{T_{2}}^{*}\right) \rho_{\text {max }}^{2}}}
\end{aligned}
$$

which results in $\Upsilon_{T_{1} \text { opt }} \approx \Upsilon_{T_{2} \text { opt }}$.

To see the impact of operating at the optimum $\Upsilon_{T}$, Fig. 1 shows $\|\mathbb{E}(P[\infty])\|$ as a function of $\Upsilon_{T}$ and for different levels of average Signal to Noise Ratio, $\Upsilon_{\text {ave }}$. For this example, Signal to Noise Ratio, $\Upsilon$, is taken to have an exponential distribution and the communication noise variance is taken as follows: $\sigma_{n}^{2}(\Upsilon)=\alpha+\delta \times \Omega(\sqrt{\Upsilon})$, where $\Omega(d)=(1 / \sqrt{2 \pi}) \int_{d}^{\infty} e^{-t^{2} / 2} d t$ for an arbitrary $d$. This is the variance of the communication noise for a binary modulation system that utilizes gray coding [10]. The following parameters are chosen for this example: $A=\left(\begin{array}{ccc}2 & 0.3 & 0.45 \\ 0.4 & 0.2 & 0.5 \\ 1.5 & 0.6 & 0.34\end{array}\right), Q=q I_{3}, C=\varsigma I_{3}, q=0.001$, $\varsigma=2, \alpha=1.27 \times 10^{-4}$ and $\delta=533.3$ (which corresponds to 10 bits per sample and quantization step size of 0.0391). It can be seen from Fig. 1 that if $\Upsilon_{T}$ is too low, estimation performance degrades due to excessive communication noise. On the other hand, having $\Upsilon_{T}$ too high will result in loss of information, which will degrade the performance. The optimum $\Upsilon_{T}$ (as predicted by Theorem 1) provides the necessary balance between loss of information and communication noise, reaching the minimums of the estimation error curves. As $\Upsilon_{T}$ increases, the estimation will approach the instability regions, predicted by (15) due to high information loss.

Remark 3: Equation (18) is derived for symmetric $A$ matrices. Still, the minimums of the curves in Fig. 1 (optimum $\Upsilon_{T}$ ), which are plotted for a non-symmetric $A$, satisfy (18). This suggests that a similar expression could be valid for the general case.

To see the impact of minimizing the determinant of the estimation error covariance, Fig. 2 shows optimum packet drop threshold as a function of average Signal to Noise Ratio, for both cases of minimizing the norm and the determinant, and for the parameters of Fig. 1. In this case, $\left|\rho_{1}\right| /\left|\rho_{2}\right|=6.3,\left|\rho_{1}\right| /\left|\rho_{3}\right|=7.7$ for matrix $A$. It can be seen that optimum points of operation are close. To see the effect of $Q$ on the optimum packet drop threshold, Fig. 3 shows asymptotic norm of the average estimation error covariance as a function of the threshold, for different $q$ and at $\Upsilon_{\text {ave }}=20 \mathrm{~dB}$. It can be seen that as the process noise increases, $\Upsilon_{T, \text { opt }}$ decreases, as expected. For instance, at $q=10$, the receiver should keep all the packets as process noise is the dominant noise.

Remark 4: In practice, (18) [or (20)] will be implemented in the physical layer. This can be done by incrementally increasing or decreasing the current packet drop threshold. Physical layer can learn the current packet loss rate statistically and can estimate the communication noise variance based on the estimate of received Signal to Noise Ratio (see [10]). It also needs to know the parameters of the state under 


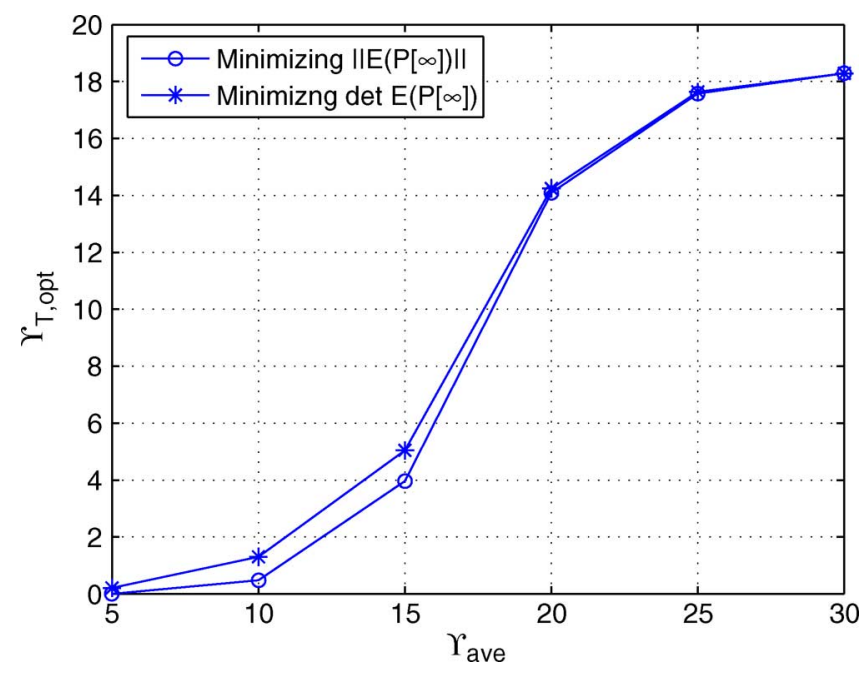

Fig. 2. Optimum packet drop threshold as a function of average Signal to Noise Ratio (in $\mathrm{dB}$ ) using two performance metrics: norm and determinant.

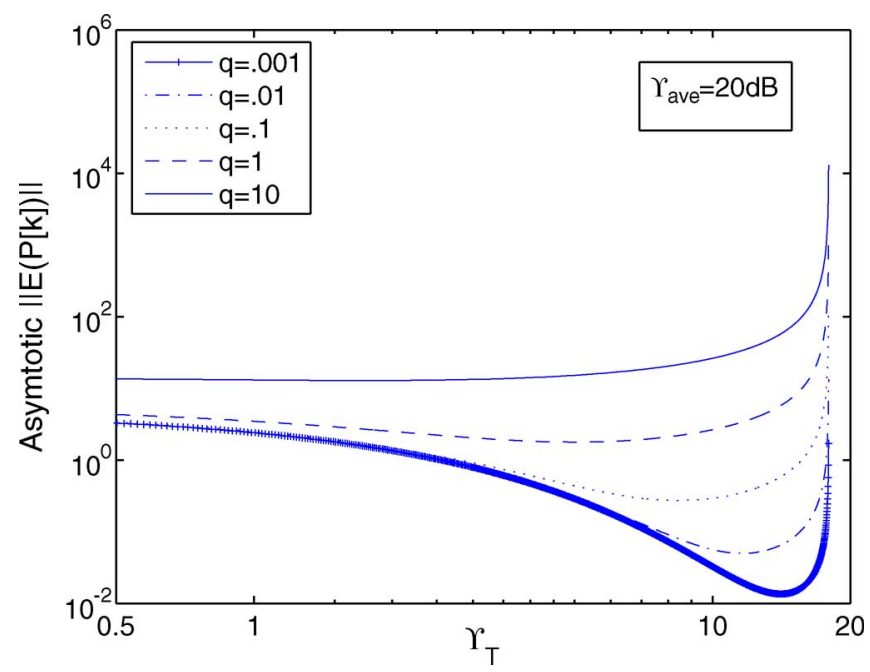

Fig. 3. Impact of $Q$ on the optimum packet drop criteria.

estimation, which are not time-varying and can therefore be passed on to it from the application layer once at the beginning of the operation. Then it increases or decreases the threshold periodically, based on the new estimates of packet loss rate and communication noise variance, in order to minimize the difference between the left-hand side and the right-hand side of (18) [or (20)].

\section{CONCLUSION}

In this technical note we considered estimation over mobile communication channels using a Kalman filter where we presented a new receiver design paradigm. We showed that dropping all the received erroneous packets is not suitable for real-time networked control applications. We then derived the optimum packet drop mechanism and proved it to be the one that balances information loss and communication noise. This means that some erroneous packets should be kept in the receiver to minimize the information loss.
There are several possible extensions for this work. For instance, we made the assumption that matrix $C$ is invertible. Furthermore, in Theorem 1 we assumed symmetric $A$ matrices. We are currently working on relaxing these assumptions. We considered one transmitter and one receiver in this technical note. The analysis and results of this technical note can be extended to scenarios with multiple sensors by replacing Signal to Noise Ratio by Signal to Interference and Noise Ratio. In this technical note we considered the impact of a wireless fading channel on the performance of a Kalman filter. Intuitively, the derived design strategy should be applicable when considering the performance of a controller. Proving this analytically is among possible extensions of this work.

\section{REFERENCES}

[1] C. Chong and S. Kumar, "Sensor networks: Evolution, opportunities and challenges," Proc. IEEE, vol. 91, no. 8, pp. 1247-1256, Aug. 2003.

[2] R. Murray, "Control in an information rich world," in Report of the Panel on Future Directions in Control, Dynamics, and Systems. Philadelphia, PA: SIAM, 2003.

[3] W. Wong and R. Brockett, "Systems with finite communication bandwidth constraints—Part I: State estimation problems," IEEE Trans. Automat. Control, vol. 42, no. 9, pp. 1294-1299, Sep. 1997.

[4] D. Goodman, Wireless Personal Communications Systems, ser. The Addison-Wesley Wireless Communications Series. Reading, MA: Addison-Wesley, 1997.

[5] M. Micheli and M. I. Jordan, "Random sampling of a continuous-time dynamical systems," in Proc. 15th Int. Symp. Math. Theory Networks Syst. (MTNS), South Bend, IN, 2002, [CD ROM].

[6] B. Sinopoli, L. Schenato, M. Franceschetti, K. Poolla, M. Jordan, and S. Sastry, "Kalman filtering with intermittent observations," in Proc. 42nd IEEE Conf. Decision Control, Dec. 9-12, 2003, vol. 1, pp. 701-708.

[7] T. S. Rappaport, Wireless Communications, Principles and Practice. Englewood Cliffs, NJ: Prentice-Hall, Jul. 1999.

[8] D. Son, B. Krishnamachari, and J. Heidemann, Experimental Analysis of Concurrent Packet Transmissions in Low-Power Wireless Networks USC-ISI Tech. Rep. ISI-TR-2005-609, Nov. 2005.

[9] T. Kailath, A. H. Sayed, and B. Hassibi, Linear Estimation, ser. Information and System Sciences. Englewood Cliffs, NJ: Prentice Hall.

[10] Y. Mostofi and R. Murray, "Effect of time-varying fading channels on the control performance of a mobile sensor node," in Proc. IEEE 1st Int. Conf. Sensor Ad hoc Commun. Networks (SECON), Santa Clara, CA, Oct. 2004, pp. 317-324. 